\title{
Relationship between Repeated Sprint Ability and Aerobic Fitness in College Volleyball Players
}

\author{
Selcen Korkmaz Eryılmaz ${ }^{1, *}$, Kerimhan Kaynak ${ }^{2}$ \\ ${ }^{1}$ Department of Coaching Education, School of Physical Education and Sports, Çukurova University, Turkey \\ ${ }^{2}$ Department of Coaching Education, Faculty of Sport Science, Erciyes University, Turkey
}

Copyright $(2019$ by authors, all rights reserved. Authors agree that this article remains permanently open access under the terms of the Creative Commons Attribution License 4.0 International License

\begin{abstract}
The main purpose of this study was to investigate the relationship between physiological variables related to aerobic fitness and repeated sprint ability (RSA) in college volleyball players. Sixteen male volleyball players $(21.1 \pm 1.4$ years $)$ took part in the study. Players first performed an incremental treadmill run test to determine their maximal oxygen uptake $\left(\mathrm{VO}_{2 \max }\right)$, ventilatory threshold (VT), peak incremental test speed and time to exhaustion. After at least 48 hours, subjects performed an RSA test consisting of $10 \times 20$-m sprints interspersed with 20 seconds of active recovery, to determine the mean sprint time (RSAmean), the best sprint time (RSAbest) and the fatigue index (FI). RSAmean was significantly negatively correlated with $\mathrm{VO}_{2 \max }(\mathrm{r}=-0.73$, $\mathrm{p}<0.01)$, VT $(\mathrm{r}=-0.62, \mathrm{p}<0.05)$, peak incremental test speed $(\mathrm{r}=-0.66, \mathrm{p}<0.01)$ and time to exhaustion $(\mathrm{r}=$ $-0.67, \mathrm{p}<0.01$ ). No relationships were found between any variables related to aerobic fitness obtained from incremental treadmill test and RSAbest and FI ( $p>0.05)$. The results of this study demonstrated that high $\mathrm{VO}_{2 \max }$ as well as VT has an important contribution to performance during repeated-sprint efforts. These findings suggest that training practices for the development of aerobic components may improve also RSA.
\end{abstract}

Keywords Maximal Oxygen Uptake, Ventilatory Threshold, Intermittent Exercise, Performance, Team Sports

\section{Introduction}

The ability of an athlete to recover quickly and maintain maximal effort during subsequent sprints is an important fitness requirement that has been termed repeated sprint ability (RSA) [1]. Repeated sprint ability is associated with superior performance in intermittent team sports [2, 3] The recovery ability between bouts of sprints may be a key determinant of performance during repeated sprinting. The maintenance of performance in the subsequent sprints primarily depends on the ability of the muscle to resynthesize the phosphocreatine $(\mathrm{PCr})$ during recovery times [4]. Sprint distances, number of sprints and recovery duration can be an important factor in determining the rate of $\mathrm{PCr}$ regeneration during and post-repeated sprint exercise $[5,6]$. During a single short sprint $(<10 \mathrm{~s})$, the contribution of aerobic metabolism to ATP resynthesis is considered insignificant. However, when this sprints is repeated at short recovery intervals $(<30 \mathrm{~s})$, the relative contribution of aerobic sources to the total energy expenditure increases with time and therefore may be important to maintain performance [7, 8]. RSA is closely related to oxidative capacity for $\mathrm{PCr}$ recovery, lactatehydrogen ions $\left(\mathrm{H}^{+}\right)$buffering, removal of intracellular inorganic phosphate $(\mathrm{Pi})$, and $\mathrm{Na}^{+} / \mathrm{K}^{+}$transport capacity [9].

The aerobic energy system plays a key role in the return to homeostasis during the rest intervals of repeated sprints [6]. Therefore, it is thought that a high level of aerobic capacity enhance the ability to recover from repeated high intensity exercise [10]. $\mathrm{VO}_{2 \max }$ is considered to be the best determinant of aerobic capacity [11]. Some studies reported a significant relationship between $\mathrm{VO}_{2 \max }$ and RSA [10, 12-15]. However, other researchers have reported a lack of correlation between RSA and $\mathrm{VO}_{2 \max }[13$, $16,17]$. A possible reason for discrepancies in these studies may be explained by differences in the variables of the protocol.

In order to improve RSA, athletes and coaches generally use training methods that include brief maximum intensity sprint repetitions of varying duration interspersed with short recovery periods. This type of repeated sprint training has been shown to improve $\mathrm{VO}_{2 \max }$ as well as anaerobic performance [18, 19]. Since resting times in the RSA protocols are very short, there is the assumption that a higher aerobic capacity will lead to faster recovery and therefore improved RSA [20]. Confirming this expectation is crucial to creating an effective training program in intermittent team sports. Although the contribution of RSA to team sport performance is generally accepted $[9,21]$, it is seen that the variables related to superior RSA have not 
yet been sufficiently clarified.

Maximum oxygen uptake $\left(\mathrm{VO}_{2 \max }\right)$ and anaerobic threshold (AT) are the most important physiological variables associated with aerobic fitness [22]. During incremental exercise, the anaerobic threshold (AT) is referred to as the ventilatory threshold (VT) if estimated by non-invasive gas exchange measurements as an alternative to measurements of the blood lactate concentration (lactate threshold) $[23,24]$. The determination of VT is based on the nonlinear increase in ventilation and carbon dioxide production due to the bicarbonate buffering of $\mathrm{H}^{+}$in response to the increase in blood lactate above resting values [24] (Wasserman, 1984). To date, many studies have examined the relationship RSA and various aerobic parameters such as maximum aerobic velocity, $\mathrm{VO}_{2 \max }$, lactate threshold and $\mathrm{VO}_{2}$ kinetic [13,25-27]. However, to our knowledge, there is no previous study examining the relationship between ventilatory threshold and RSA. In addition, to our knowledge, there are no studies investigating the relationship between aerobic fitness and RSA in volleyball players. The purpose of this study was to investigate the relationship between physiological variables related to aerobic fitness and RSA in college volleyball players.

\section{Materials and Methods}

\subsection{Subjects and Experimental Design}

Sixteen male amateur volleyball players (mean \pm SD; age $21.1 \pm 1.4$ years, height $183.3 \pm 4.9 \mathrm{~cm}$, weight $73.5 \pm$ $8.1 \mathrm{~kg}$ ) competing in Division I of the Turkish University League volunteered to participate in the study. All of the subjects were members of the Erciyes University volleyball team. All players had trained and competed regularly in volleyball for at least 4 years. The Erciyes University Ethics Committee approved the study (2016/651). All testing and training procedures were fully explained, and written informed consent was obtained for each subject. The study lasted a total of one week and started 2 weeks after the beginning of the preparatory training season. All players performed two tests with an interval of 2 days between each test. The two tests consisted of an incremental treadmill test and the repeated sprint test. Tests began with an incremental treadmill test and took place at the High Altitude and Sports Science Research and Implementation Center Laboratory at Erciyes University. All repeated sprint testing took place on an indoor volleyball court at the Erciyes University School of Physical Education and Sport. In order to prevent unnecessary fatigue accumulation, the players were not allowed to perform any training the day before each testing.

\subsection{Incremental Treadmill Test}

The maximal oxygen uptake $\left(\mathrm{VO}_{2 \max }\right)$ and ventilatory threshold (VT) were determined from a progressive intensity and continuous effort treadmill protocol. All tests were performed on a motorized treadmill $(\mathrm{h} / \mathrm{p} /$ Cosmos Quasar med, Nussdorf-Traunstein, Germany). Oxygen uptake $\left(\mathrm{VO}_{2}\right)$, carbon dioxide output $\left(\mathrm{VCO}_{2}\right)$ and minute ventilation (VE) were measured on-line using a breath-by-breath cardiopulmonary exercise testing system (Quark PFT Ergo, Cosmed Srl, Rome, Italy). Before each test, ambient conditions were measured, and the gas analyzers and turbine flowmeter were calibrated with known certified gas concentrations $\left(16 \% \mathrm{O}_{2}, 5 \% \mathrm{CO}_{2}\right.$, and balanced $\mathrm{N}_{2}$ ) and a $3 \mathrm{~L}$ calibration syringe, respectively, following the manufacturer's instructions. During the incremental testing period, heart rate (HR) was monitored continuously using a wireless HR monitor (S610i, Polar, Finland) and was synchronized to ventilatory signals.

To make sure the players were properly warmed up, prepared, and accustomed to the treadmill, each participant had to warm up for 6 min at their own pace. Then the players were allowed to stop and stretch for about $3 \mathrm{~min}$. Following the warm-up, players started running at $7 \mathrm{~km} / \mathrm{h}$ with speed increments of $1 \mathrm{~km} / \mathrm{h}$ every minute until they could no longer keep pace. The players were instructed to run until voluntary exhaustion and were given strong verbal encouragement throughout the test to elicit their best performance. Achievement of $\mathrm{VO}_{2 \max }$ was considered as the attainment of at least two of the following criteria: 1) a plateau in $\mathrm{VO}_{2}$ despite increasing speed, 2) a respiratory exchange ratio $\left(\mathrm{VCO}_{2} / \mathrm{VO}_{2}\right)$ above 1.10 , and 3$)$ a $\mathrm{HR}$ (heart rate) within 10 beats per minute of age-predicted maximum HR (220 - age) [28]. The maximal oxygen uptake value was expressed as a relative value (milliliters per minute per body mass; $\mathrm{ml} / \mathrm{kg} / \mathrm{min}$ ).

Breath-by-breath $\mathrm{VO}_{2}$ was smoothed using a five-step average filter and then reduced to $15 \mathrm{~s}$ stationary averages for the incremental test (Data Management Software, Cosmed, Rome, Italy) to reduce the noise so as to enhance the underlying characteristics. The $\mathrm{VO}_{2 \max }$ was defined as the highest $15 \mathrm{~s} \mathrm{VO}_{2}$ value reached during the incremental test. Time to exhaustion was recorded as the time from the start of the run until the point of exhaustion (the time at which the subject could no longer maintain the pace of the treadmill). The VT was determined using the V-slope method described by Beaver et al. [23]. The VT was defined as the $\mathrm{VO}_{2}$ value corresponding to the intersection of two linear regression lines derived separately from the data points below and above the breakpoint in the $\mathrm{VCO}_{2}$ versus the $\mathrm{VO}_{2}$ relationships. Linear regression analyses were performed by using the Sigma Plot program (Sigma Plot 12.0, Systat Software Inc., Chicago, USA).

\subsection{Repeated Sprint Ability Test}

The repeated sprint ability test (RSA) consisted of ten repetitions of $20 \mathrm{~m}$ all-out sprints, interspersed with $20 \mathrm{~s}$ of active recovery, during which the players jogged back to the starting line. Sprint times were measured for each $20 \mathrm{~m}$ sprint with two ports of light sensors (Newtest 2000-sprint 
timing system; Newtest Oy, Oulu, Finland) placed at the starting line and the finishing line, and the recovery time was controlled by a hand-held stopwatch. RSA was performed after a standard warm-up procedure that included $5 \mathrm{~min}$ of jogging, $5 \mathrm{~min}$ of stretching and $5 \mathrm{~min}$ of specific running drills at increasing speed. After the warm-up, each player performed two preliminary $20 \mathrm{~m}$ maximal sprints separated by a 2 min recovery period. The fastest sprint time was used as the criterion score for the RST. After these trials, subjects rested for $5 \mathrm{~min}$ before the start of the RST. If the performance in the first sprint of the RST was worse than the criterion score (i.e., an increase in time greater than $2.5 \%$ ), the test was immediately terminated and subjects were required to repeat the RST test with maximum effort after a 5-min rest [19]. The players stood $30 \mathrm{~cm}$ behind the start line to avoid premature triggering of the timing system and completed $10 \times 20 \mathrm{~m}$ sprints with $20 \mathrm{~s}$ of active jogging recovery between sprints. During the $20 \mathrm{~s}$ active recovery period, the players were required to decelerate after passing the finish line, and to jog around a cone and return to the starting line. A cone was placed $5 \mathrm{~m}$ beyond the finish line, and in this way the players had a chance to decelerate their speed. Players were instructed to complete all sprints as fast as possible, and strong verbal encouragement was provided to each player during all sprints. The recovery time was controlled by a hand-held stopwatch. During the active recovery, continuous verbal feedback was provided to ensure the players had returned to the start position in time to begin the next sprint.

From the RST data, the best sprint time (RSA best; the fastest $20 \mathrm{~m}$ sprint time), mean sprint time (RSA mean; mean time to complete 10 sprints) and the fatigue index expressed as a percentage were selected for the analysis. The fatigue index (FI) was used as an indication of fatigue and was calculated according to Fitzsimmons et al. [29].

$\mathrm{FI}=100 \times($ total sprint time $\div$ ideal sprint time $)-100$, where total sprint times $=$ sum of sprint times from all sprints, and ideal sprint time $=$ number of sprints $\times$ best sprint time.

\subsection{Statistical Analysis}

Data are reported as means \pm standard deviation (SD). Statistical significance was accepted at $\mathrm{p}<0.05$. The normality distribution of the data was checked with the Shapiro-Wilk test. A Pearson product-moment correlation was used to determine the relationship between aerobic fitness and RSA variables. Magnitude of correlation was qualitatively ranked according to Hopkins et al. [30] as follows: $\mathrm{r} \leq 0.1$, trivial; $0.1<\mathrm{r} \leq 0.3$, small; $0.3<\mathrm{r} \leq 0.5$, moderate; $0.5<\mathrm{r} \leq 0.7$, large; $0.7<\mathrm{r} \leq 0.9$, very large; and $r>0.9$, almost perfect. Simple linear regression analysis was used to determine the success of prediction. Confidence intervals $(95 \% \mathrm{CI})$ were calculated for each correlation.

\section{Results}

The physiological characteristics of the 16 volleyball players evaluated in present study are summarized in Table 1. RSA mean was significantly negatively correlated with the $\mathrm{VO}_{2 \max }(\mathrm{r}=-0.73, \mathrm{p}=0.01)$, the VT $(\mathrm{r}=-0.62, \mathrm{p}=$ $0.01)$, the peak incremental test speed $(r=-0.66, p=0.005)$ and the time to exhaustion $(r=-0.67, p<0.004)$ (Figure 1). There was no correlation between the RSA best and the $\mathrm{VO}_{2 \max }(\mathrm{r}=-0.41, \mathrm{p}=0.1)$, the VT $(\mathrm{r}=-0.41, \mathrm{p}=0.11)$, the peak incremental test speed $(r=-0.44, p=0.08)$, the time to exhaustion $(r=-0.48, p=0.057)$. There was no correlation between the FI and the $\mathrm{VO}_{2 \max }(\mathrm{r}=-0.43, \mathrm{p}=$ $0.09)$, the VT $(r=-0.2, p=0.4)$, the peak incremental test speed $(r=-0.7, p=0.7)$, the time to exhaustion $(r=-0.59$, $\mathrm{p}<0.8)$.

Table 1. Descriptive statistics of the aerobic fitness and repeated-sprint variables

\begin{tabular}{ccccccc}
\hline $\mathrm{VO}_{2 \max }$ & VT & Time to ex & Speed $_{\text {peak }}$ & RSA best & RSA mean & FI (\%) \\
\hline $50.3 \pm 3.5$ & $38.3 \pm 5.4$ & $8.9 \pm 1.2$ & $15.5 \pm 1.3$ & $2.97 \pm 0.1$ & $3.21 \pm 0.1$ & $8.04 \pm 2.7$ \\
\hline
\end{tabular}

Values are mean \pm standard deviation. $\mathrm{VO}_{2 \max }=$ maximal oxygen uptake $\left(\mathrm{ml} \mathrm{kg}^{-1} \mathrm{~min}^{-1}\right), \mathrm{VE}=$ ventilatory threshold $\left(\mathrm{ml} \mathrm{kg}^{-1} \mathrm{~min}^{-1}\right)$, Time to ex $=$ time to exhaustion $(\mathrm{min})$, Speed $_{\text {peak }}=$ peak incremental test speed $(\mathrm{km} \mathrm{h}-1)$, RSA best = best sprint time $(\mathrm{s})$, RSA mean $=$ mean sprint time $(\mathrm{s})$, FI $=$ fatigue index 

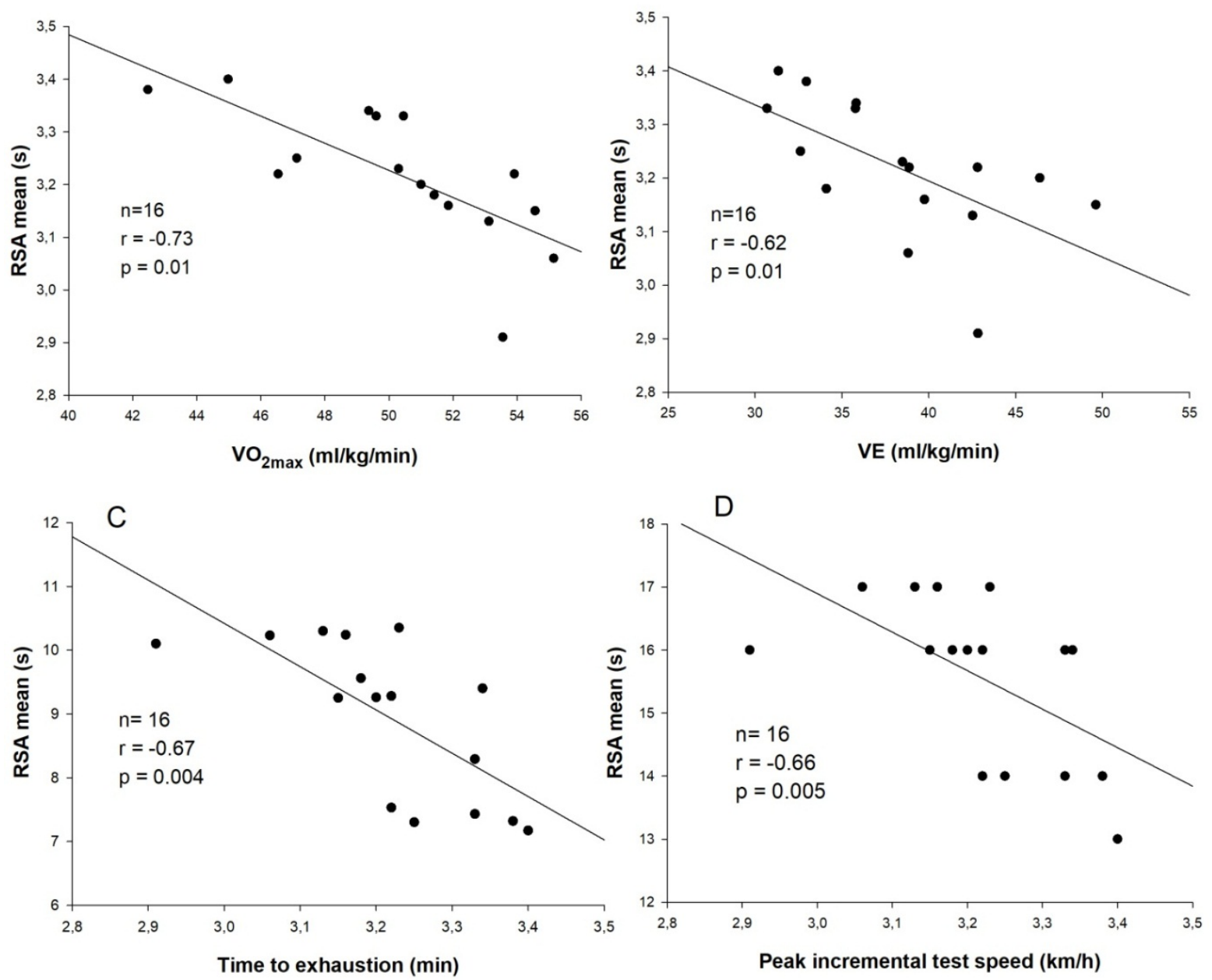

Figure 1. Relationship between $\mathrm{A})$ maximal oxygen uptake $\left(\mathrm{VO}_{2 \max }\right)$ and mean sprint time (RSA mean), B) ventilatory threshold (VT) and mean sprint time (RSA mean), C) time to exhaustion and mean sprint time (RSA mean), and D) peak incremental test speed and mean sprint time (RSA mean) in all subjects.

\section{Discussion}

The purpose of the present study was to examine the relationship between aerobic fitness and RSA in college volleyball players. The determinants of aerobic fitness in the present study were considered as $\mathrm{VO}_{2 \max }$, VT, peak incremental test speed and time to exhaustion. Our results showed that RSA mean was significantly correlated with $\mathrm{VO}_{2 \max }$, VT, peak incremental test speed and time to exhaustion. No relationship however was observed between any variables related to aerobic fitness and RSA best and FI. Our results confirm the association of good aerobic fitness with RSA. In order to enhance the repeated high intensity exercise performance of players, it may be important to include sports-specific training for the development of both aerobic and anaerobic components in the annual training cycle.

High aerobic capacity is considered to be a prerequisite for superior anaerobic performances during intermittent activities [31]. $\mathrm{VO}_{2 \max }$ is the most widely accepted criteria for assessing aerobic capacity [11]. The main finding of present study was that the high negative correlation was between $\mathrm{VO}_{2 \max }$ and RSA mean $(r=-0.736, P<0.01)$. Consistent with our results, previous studies have reported that there is a significant relationship between $\mathrm{VO}_{2 \max }$ and RSA [10,12-15]. On the other hand, this contrasts with the results of some studies [13, 16, 17]. In contrast to our study, some studies have found that the relationship between $\mathrm{VO}_{2 \max }$ and only fatigue index of RSA assessments [32-34]. Previous studies focusing on the relationship of $\mathrm{VO}_{2 \max }$ to RSA have used different repeated sprint test protocols (e.g. sprint distances, number of sprints, recovery periods). Our finding is in consistent with the results reported by Sanders et al. [12] that $\mathrm{VO}_{2 \max }$ was largely correlated with mean sprint time for ten $30 \mathrm{~m}$ sprints interspersed with $30 \mathrm{~s}(\mathrm{r}=$ $-0.767)$. In a study involving professional soccer players a moderate relationship $(r=-0.655)$ was reported between $\mathrm{VO}_{2 \max }$ and a mean sprint time of RSA protocol comprising $6 \times 40 \mathrm{~m}$ sprints with $20 \mathrm{~s}$ recovery between each sprint [15]. The results of this study supported the theory that $\mathrm{VO}_{2 \max }$ is an important factor influencing recovery from RSA in intermittent sports. Similarly a study conducted on soccer players reported a moderate relationship between the $\mathrm{VO}_{2 \max }$ and the mean sprint time of RSA protocol 
comprising $6 \times 40 \mathrm{~m}$ sprints separated by $20 \mathrm{~s}$ recoveries $(\mathrm{r}$ $=-0.45$ ) [14]. Furthermore in a study involving field hockey and soccer players a small relationship was observed between the $\mathrm{VO}_{2 \max }$ and the total sprint time for eight $40 \mathrm{~m}$ sprints interspersed with $30 \mathrm{~s}$ recovery $(\mathrm{r}=$ -0.34 and -0.32 ) [10]. In another study involving various team-sport players, it was shown that that there was a significant relationship between $\mathrm{VO}_{2 \max }$ and fatigue index but not with total sprint time for fifteen $40 \mathrm{~m}$ sprints alternated with $25 \mathrm{~s}$ recoveries [33]. However, a study conducted on elite soccer players reported a trivial relationship between a $6 \times 40 \mathrm{~m}$ RSA assessment and the $\mathrm{VO}_{2 \max }[17]$. On the other hand, some studies using indirect methods to determine $\mathrm{VO}_{2 \max }$ using field tests have shown a relationship between $\mathrm{VO}_{2 \max }$ and RSA indices [32, 35, 36]. However, other studies have shown a weak relationship between $\mathrm{VO}_{2 \max }$ of indirectly determined and RSA indices [37, 38]. A potential reason for discrepancies in the results of these studies may be explained by differences in the type of protocol used for the repeated sprint test. The sprint distances, number of sprints and recovery duration between sprints can be considered as important factors affecting the contribution of the energy system during repeated-sprint exercise [7, 8].

It is generally believed that $\mathrm{VO}_{2 \max }$ is not associated with RSA when distances less than $40 \mathrm{~m}$ (or $6 \mathrm{~s}$ ) are used [13, 20]. A study by Aziz et al. [16] supporting this idea has shown that $\mathrm{VO}_{2 \max }$ was not associated with any of the performance indices in the RSA protocol that consisted of six, $20 \mathrm{~m}$ sprints interspersed with $20 \mathrm{~s}$ of active recovery. They suggested that the $20 \mathrm{~m}$ sprint distance may cause the PCr storages to be depleted less than previous studies using longer sprint duration (e.g. $>6 \mathrm{~s}$ ). On the other hand, sprint distances of $20 \mathrm{~m}$ in their studies are composed of six repetitions that are lower than our study. In the present study, as the number of $20 \mathrm{~m}$ sprints was ten, this may explain that the correlation between RSA and $\mathrm{VO}_{2 \max }$ is higher than their study. The number of sprints may affect this relationship between RSA and $\mathrm{VO}_{2 \max }$ by altering the contribution of the aerobic energy system. Repeated sprints of a more number may require a greater contribution from aerobic metabolism, especially during subsequent sprints [7]. Merkel et al. [17] have suggested that the aerobic system is more related to power maintenance in a high number of short sprint repetitions $(12 \times 20 \mathrm{~m})$ than low number of long sprint repetitions $(6 \times 40 \mathrm{~m})$. A possible explanation of the discrepancy in the results presented in the different studies may be the number of the sprint used in the RSA protocol. RSA protocol with short sprint distance may be advantageous in terms of reflecting physical activity during team sport matches. It may be better to evaluate the relationship between aerobic capacity and RSA by using test protocols including shorter distance and more sprint repetitions.

The relationship between $\mathrm{VO}_{2 \max }$ and mean sprint time $(\mathrm{r}=\neg 0.736)$ in the present study is stronger than the results of previous studies $[10,13,14]$. It has been suggested that the relationship between aerobic fitness and RSA may be stronger in untrained to moderately trained subjects than well-trained team sports athletes [25]. However, it is seen that these researchers determine the training levels of the subjects according to the $\mathrm{VO}_{2 \max }$ values. In the present study, as aerobic capacity is not constitute a priority for sports-specific fitness requirements in volleyball, players were primarily trained with anaerobic exercises. Therefore, volleyball players have low $\mathrm{VO}_{2 \max }$ values compared to other team sports such as soccer.

Our results are consistent with the results reported by other studies that no aerobic fitness variable was associated with the best sprint time during the RSA test $[10,13,14]$. A single sprint is carried out mainly via anaerobic pathways, but it has been shown that the relative contribution of aerobic metabolism to the total energy provision increases when the exercise bout is repeated with short recovery intervals [7, 8]. In our study, the lack of correlation between the best $20 \mathrm{~m}$ sprint time and aerobic capacity may be due to the ineffective of the aerobic energy system in the energy demands of a single sprint.

In the present study, $\mathrm{VO}_{2 \max }$ was not used as the sole indicator of aerobic fitness in order to evaluate the relationship between aerobic fitness and RSA. Significant negative correlation in this study was found between VT and RSA mean. VT has been reported to be significantly associated with muscle oxidative capacity $[39,40]$. Some studies have been shown that RSA indices are correlated with velocity at the onset of blood-lactate accumulation [13] and lactate threshold [25]. On the other hand, another study showed no significant relationship between RSA indices and velocity at lactate threshold [26]. However, to the best of our knowledge, there is no study investigating the relationship between VT and RSA in the literature. The results of our study showed that in addition to $\mathrm{VO}_{2 \max }$, VT may also be contributing performance during repeated-sprint efforts.

Another finding of this study is that the RSA mean was significantly correlated with the maximal running speed and time to exhaustion. RSA indices have been shown to correlate better with peak incremental test speed than with $\mathrm{VO}_{2 \max }$ [27]. Da Silvathe et al. [13] demonstrated that mean time for 7 sprints of $34.2 \mathrm{~m}$ is more strongly correlated with the minimum velocity needed to reach $\mathrm{VO}_{2 \max }$ than $\mathrm{VO}_{2 \max }$. Papadakis et al. [26] shown that mean time for 6 sprints of $35 \mathrm{~m}$ correlated with velocity at $\mathrm{VO}_{2 \max }$ but not with $\mathrm{VO}_{2 \max }$. Similarly, Cipryan and Gajda [37] implemented a RSA test consisting of six $35 \mathrm{~m}$ sprints and found that performance indices in the RSA test are associated with peak power in the graded treadmill test but not with $\mathrm{VO}_{2 \max }$.

\section{Conclusions}

The present study indicated that repeated sprint ability using a $10 \times 20 \mathrm{~m}$ field testing protocol departing every $20 \mathrm{~s}$ in volleyball players is closely related to aerobic fitness. In conclusion, physiological variables related to aerobic fitness such as $\mathrm{VO}_{2 \max }$ and VT has an important 
contribution to performance during repeated-sprint efforts. This suggests that training practices for the development of aerobic components may improve also RSA.

\section{Conflict of Interest}

The authors declare no conflict of interest.

\section{Funding}

This research received no funding.

\section{REFERENCES}

[1] D. Bishop, M. Spencer, R. Duffield, S. Lawrence. The validity of a repeated sprint ability test, Journal of Science and Medicine in Sport, Vol.4, No.1, 19-29, 2001.

[2] E. Rampinini, D. Bishop, S. M. Marcora, D. Ferrari Bravo, R. Sassi, F. M. Impellizzeri. Validity of simple field tests as indicators of match-related physical performance in top-level professional soccer players, International Journal of Sports Medicine, Vol.28, No.3, 228-235, 2007.

[3] F. M. Impellizzeri, E. Rampinini, C. Castagna, D. Bishop, D. Ferrari Bravo, A. Tibaudi, U. Wisloff. Tibaudi A and Wisloff U. Validity of a repeated-sprint test for football, International Journal of Sports Medicine, Vol.29, No.11, 899-905. 2008.

[4] S. McMahon, D. Jenkins. Factors affecting the rate of phosphocreatine resynthesis following intense exercise, Sports Medicine, Vol.32, No.12, 761-784. 2002.

[5] B. Dawson, C. Goodman, S. Lawrence, D. Preen, T. Polglaze, M. Fitzsimmons, P. Fournier. Muscle phosphocre atine repletion following single and repeated short sprint effort, Scandinavian journal of Medicine and Science in Sports, Vol.7, No.4, 206-213. 1997.

M. Glaister. Multiple sprint work: physiological responses, mechanisms of fatigue and the influence of aerobic fitness, Sports Medicine, Vol.35, No.9, 757-777. 2005.

[6] G. C. Gaitanos, C. Williams, L.H. Boobis, S. Brooks. Human muscle metabolism during intermittent maximal exercise, Journal of Applied Physiology, Vol.75, No.2, 712-719. 1993.

[7] P. D. Balsom, G. C. Gaitanos, B. Ekblom, B. Sjodin. Reduced oxygen availability during high intensity intermittent exercise impairs performance, Acta Physiologica Scandinavica, Vol.152, No.3, 279-285. 1994.

[8] O. Girard, A. Mendez-Villanueva, D. Bishop. Repeated-sprint ability - part I: factors contributing to fatigue, Sports Medicine, Vol.41, No.8, 673-694. 2011.

[9] A. Aziz, M. Chia, K. The. The relationship between maximal oxygen uptake and repeated sprint performance indices in field hockey and soccer players, Journal of sports medicine and physical fitness, Vol.40, No.3, 195-200. 2000.
[10] G. Rankovic, V. Mutavdzic, D. Toskic, A. Preljevic, M. Kocic, G. Nedin Rankovic, N. Damjanovic. Aerobic capacity as an indicator in different kinds of sports, Bosnian Journal of Basic Medical Sciences, Vol.10, No.1, 44-48. 2010.

[11] G. J. Sanders, Z. Turner, B. Boos, C. A. Peacock, W. Peveler, A. Lipping. Aerobic capacity is related to repeated sprint ability with sprint distances less than 40 meters, International Journal of Exercise Science. Vol.10, No.2, 197-204. 2017.

[12] J. F. Da Silva, L. G. A. Guglielmo, D. Bishop. Relationship between different measures of aerobic fitness and repeated sprint ability in elite soccer players, Journal of strength and conditioning research, Vol.24, No.8, 2115-2121. 2010.

[13] E. Rampinini, A. Sassi, A. Morelli, S. Mazzoni, M. Fanchi ni, A. J. Coutts. Repeated-sprint ability in professional and amateur soccer players, Applied Physiology Nutrition and Metabolism, Vol.34, No.6, 1048-1054. 2009

[14] R. M. Jones, C. C. Cook, L. P. Kilduff, Z. Milanović, N. James, G. Sporiš, B. Fiorentini, F. Fiorentini, A. Turner, G. Vučković. Relationship between repeated sprint ability and aerobic capacity in professional soccer players, Scientific World Journal, Vol.2013, 952350. 2013.

[15] A. Aziz, S. Mukherjee, M. Chia, K. The. Relationship between measured maximal oxygen uptake and aerobic endurance performance with running repeated sprint ability in young elite soccer players, Journal of sports medicine and physical fitness, Vol.47, No.4, 401-407. 2007.

[16] Y. Meckel, O. Machnai, A. Eliakim. Relationship among repeated sprint tests, aerobic fitness, and anaerobic fitness in elite adolescent soccer players, Journal of strength and conditioning research, Vol.23, No.1, 163-169. 2009.

[17] K. Kaynak, S. Korkmaz Eryılmaz, S. Aydoğan, D. Mihailov. The effects of $20-\mathrm{m}$ repeated sprint training on aerobic capacity in college volleyball players, Biomedical Human Kinetics, Vol.9, No.1, 43-50. 2017.

[18] D. F. Bravo, F. M. Impellizzeri, E. Rampinini, C. Castagna, D. Bishop, U. Wisloff. Sprint vs. interval training in football, International Journal of Sports Medicine, Vol.29, No.8, 668-674. 2008.

[19] A. N. Turner, P. F. Stewart. Repeat sprint ability, Strength and Conditioning Journal, Vol.35, No.1, 37-41. 2013.

[20] M. Spencer, D. Bishop, B. Dawson, C. Goodman.Physiolo gical and metabolic responses of repeated-sprint activities: specific to field-based team sports, Sports Medicine, Vol.35, No.12, 1025-1044. 2005.

[21] J. Helgerud. Maximal oxygen uptake, anaerobic threshold and running economy in women and men with similar performance levels in marathons, European journal of applied physiology and occupational physiology. Vol.68, No.2, 155-61. 1994.

[22] W. L. Beaver, K. Wasserman, B. J. Whipp. A new method for detecting the anaerobic threshold by gas exchange, Journal of applied physiology, Vol.60, No.6, 2020-2027. 1986.

[23] K. Wasserman. 1984. The anaerobic threshold measurement to evaluate exercise performance, American 
review of respiratory disease, Vol.129, No. 2 Pt 2, 35-40.

[24] D. Bishop, J. Edge, C. Goodman. Muscle buffer capacity and aerobic fitness are associated with repeated-sprint ability in women, European journal of applied physiology, Vol.92, No.4-5, 540-7. 2004.

[25] L. Papadakis, M. Mitrotasios, K. Patras. Influence of aerobic endurance, sports speed and strength to repeated sprint performance, Biology of Exercise, Vol.13, No.1, 46-56. 2017.

[26] M. Buchheit. Repeated-sprint performance in team sport players: associations with measures of aerobic fitness, metabolic control and locomotor function, International journal of sports medicine, Vol.33, No.3, 230-9. 2012.

[27] E. T. Howley, D. R. Jr Bassett, H. G. Welch. Criteria for maximal oxygen uptake: review and commentary, Medicine and science in sports and exercise, Vol.27, No.9, 1292-1301. 1995.

[28] M. Fitzsimmons, B. Dawson, D. Ward, A. Wilkinson. Cycling and running tests of repeated sprint ability, Australian Journal of Science and Medicine in Sport, Vol.25, No.4, 82-87. 1993.

[29] W. Hopkins, S. Marshall, A. Batterham, J. Hanin. Progressive statistics for studies in sports medicine and exercise science, Medicine and science in sports and exercise, Vol.41, No.1, 3-13. 2009.

[30] D. Jenkins. The importance of aerobic fitness for field games players, Sports Coach, Vol.16, 22-23. 1993.

[31] Z. Gharbi, W. Dardouri, R. Haj-Sassi, K. Chamari, N. Souissi. Aerobic and anaerobic determinants of repeated sprint ability in team sports athletes, Biology of Sport, Vol.32, No.2, 207-12. 2015.

[32] G. Dupont, G. P. Millet, C. Guinhouya, S. Berthoin. Relationship between oxygen uptake kinetics and performance in repeated running sprints, European journal of applied physiology, Vol.95, No. 1, 27-34. 2005.

[33] B. Dawson, M. Fitzsimons, D. Ward. The relationship of repeated sprint ability to aerobic power and performance measures of anaerobic work capacity and power, Australian journal of science and medicine in sport, Vol.25, No.4, 88-93. 1993.

[34] N. Gibson, J. Currie, R. Johnston, J. Hill. Relationship between measures of aerobic fitness, speed and repeated sprint ability in full and part time youth soccer players, Journal of sport medicine and physical fitness, Vol.53, No.1, 9-16. 2013.

[35] M. Spencer, D. Pyne, J. Santisteban, I. Mujika. Fitness determinants of repeated-sprint ability in highly trained youth football players, International Journal of Sports Physiology and Performance, Vol.6, No.4, 497-508. 2011.

[36] L. Cipryan, V. Gajda. The influence of aerobic power on repeated anaerobic exercise in junior soccer players, Journal of Human Kinetics, Vol.28, No.-1, 63-71. 2011.

[37] D. B. Pyne, P. U. Saunders, P. G. Montgomery, A. J. Hewitt, K. Sheehan. Relationships between repeated sprint testing, speed, and endurance., Journal of strength and conditioning research, Vol.22, No.5, 1633-1637. 2008.
[38] J. L. Ivy, R. T. Withers, P. J. Van Handel, D. H. Elger, D. L. Costill. Muscle respiratory capacity and fiber type as determinants of the lactate threshold, Journal of applied physiology: respiratory, environmental and exercise physiology, Vol.48, No.3, 523-7. 1980.

[39] H. Rusko, P. Rahkila, E. Karvinen. Anaerobic threshold, skeletal muscle enzymes and fiber composition in young female cross-country skiers, Acta physiologica Scandinavica, Vol.108, No.3, 263-8. 1980. 